\title{
New Colour Transformations for the Sloan Photometry, and Revised Metallicity Calibration and Equations for Photometric Parallax Estimation
}

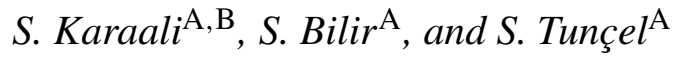 \\ A Istanbul University, Department of Astronomy and Space Sciences, 34119 University-Istanbul, Turkey \\ B Corresponding author. Email: karsa@istanbul.edu.tr
}

Received 2004 March 24, accepted 2004 July 12

\begin{abstract}
We evaluated new colour transformations for the Sloan photometry by 224 standards and used them to revise both the equations for photometric parallax estimation and metallicity calibration cited by Karaali et al. (2003). This process improves the metallicity and absolute magnitude estimations by $[\mathrm{Fe} / \mathrm{H}] \leq 0.3 \mathrm{dex}$ and $M_{g^{\prime}}^{\mathrm{H}} \geq 0.1$ mag respectively. There is a high correlation for metallicities and absolute magnitudes derived for two systems, $U B V$ and Sloan, by means of the revised calibrations.
\end{abstract}

Keywords: techniques: Sloan photometry — galaxy: abundances — stars: distances

\section{Introduction}

In a recent paper, we (Karaali et al. 2003) presented a new procedure for the photometric parallax estimation and extended it to the Sloan photometry. We also highlighted the advantage of the new procedure relative to the ones already used. In particular, we noticed that this procedure would be more appropriate for stars of a population with a large metallicity range, for which a single colourmagnitude diagram is used in the literature (cf. Chen et al. 2001; Siegel et al. 2002).

However, unlike the colour transformations derived for broad-band photometric systems (see, e.g., Buser 1978), the transformations derived for the Sloan system (Fukugita et al. 1996) are functions of a single colour, i.e. the colour $\left(u^{\prime}-g^{\prime}\right)$ is a function of only $(U-B)$, and $\left(g^{\prime}-r^{\prime}\right)$ is another function of only $(B-V)$. This was the case about a decade ago (cf. Fukugita et al. 1996), and it is still the same for the very recent years (Smith et al. 2002). We thought to evaluate new colour transformations for $\left(u^{\prime}-g^{\prime}\right)$ and $\left(g^{\prime}-r^{\prime}\right)$, which would cover both $(U-B)$ and $(B-V)$, by using the data of standards already used in the literature. This is the main scope of this work. We will show in the following Sections that such an approach provides more precise absolute magnitudes (and metallicities) than those in the paper of Karaali et al. (2003).

Also, we wish to emphasise that the new photometric parallax and $[\mathrm{Fe} / \mathrm{H}]$ equations are only one example of the improvement in science that could result from these new transformations.

In Section 2 we present the data used for calibration and the new colour transformations, and in Section 3 the new metallicity calibration is given. New equations for photometric parallaxes are given in Section 4 and in Section 5 a short conclusion is provided.

\section{New Colour Transformations for the Sloan Photometry}

We used the $U B V$ data of Landolt (1992), for 251 stars, whereas $u^{\prime} g^{\prime} r^{\prime}$ data for the same stars are provided from the CASU INT Wide Field Survey ${ }^{1}$. Figure 1, which plots $\left(g^{\prime}-r^{\prime}\right)$ versus $(B-V)$ and $\left(u^{\prime}-g^{\prime}\right)$ versus $(U-B)$, shows that there are some stars at extreme locations in these diagrams. These stars either have $(B-V)$ colour indices less than $0.3 \mathrm{mag}$ or larger than $1.1 \mathrm{mag}$, where the calibration of Karaali et al. (2003) does not hold, or their standard errors for the colour indices cited above are relatively large. The total number of stars which lie at extreme locations in Figure 1 is 27 . We excluded them from the sample and we marked the remaining 224 points with agreeable colours into Figure 2. It is interesting that the points in each Figure do not lie on a straight line, but they form a band. This shows schematically that $\left(u^{\prime}-g^{\prime}\right)$ and $\left(g^{\prime}-r^{\prime}\right)$ are functions of both $(U-B)$ and $(B-V)$.

We adopted the colour transformations as follows and evaluated the coefficients by means of the least-squares method:

$$
\begin{aligned}
g^{\prime}-r^{\prime} & =a(B-V)+b(U-B)+c \\
u^{\prime}-g^{\prime} & =d(U-B)+e(B-V)+f
\end{aligned}
$$

Thus, we obtained the new colour transformations for the Sloan photometry as in the following:

$$
\begin{aligned}
g^{\prime}-r^{\prime} & =1.023(B-V)+0.016(U-B)-0.187 \\
u^{\prime}-g^{\prime} & =0.779(U-B)+0.755(B-V)+0.801
\end{aligned}
$$

We recall the colour transformations of Fukugita et al. (1996) which were used in the paper of Karaali et al. (2003)

\footnotetext{
${ }^{1}$ http://www.ast.cam.ac.uk/ $\sim$ wf csur/index.php
} 

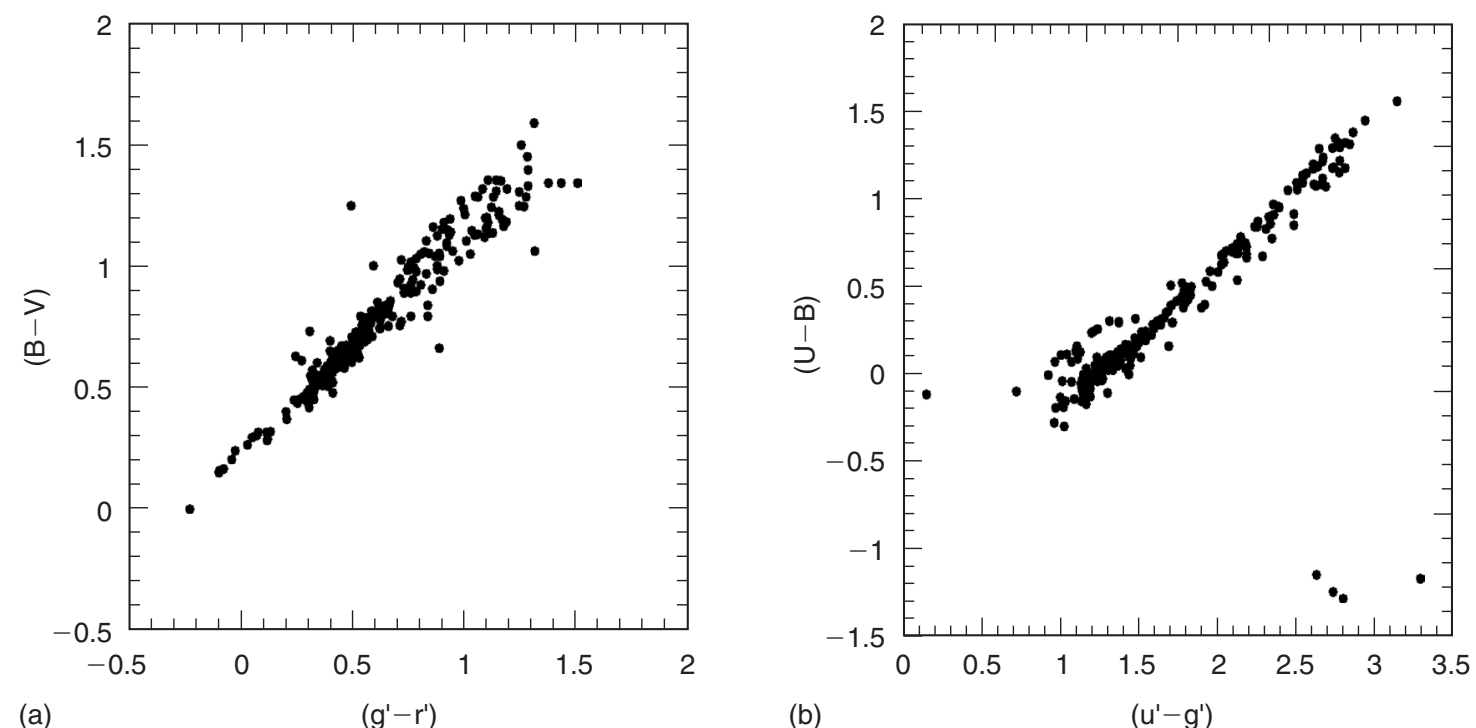

Figure 1 (a) $\left(g^{\prime}-r^{\prime}\right)$ versus $(B-V)$ and (b) $\left(u^{\prime}-g^{\prime}\right)$ versus $(U-B)$ for the original sample of stars. There are some stars at extreme locations.
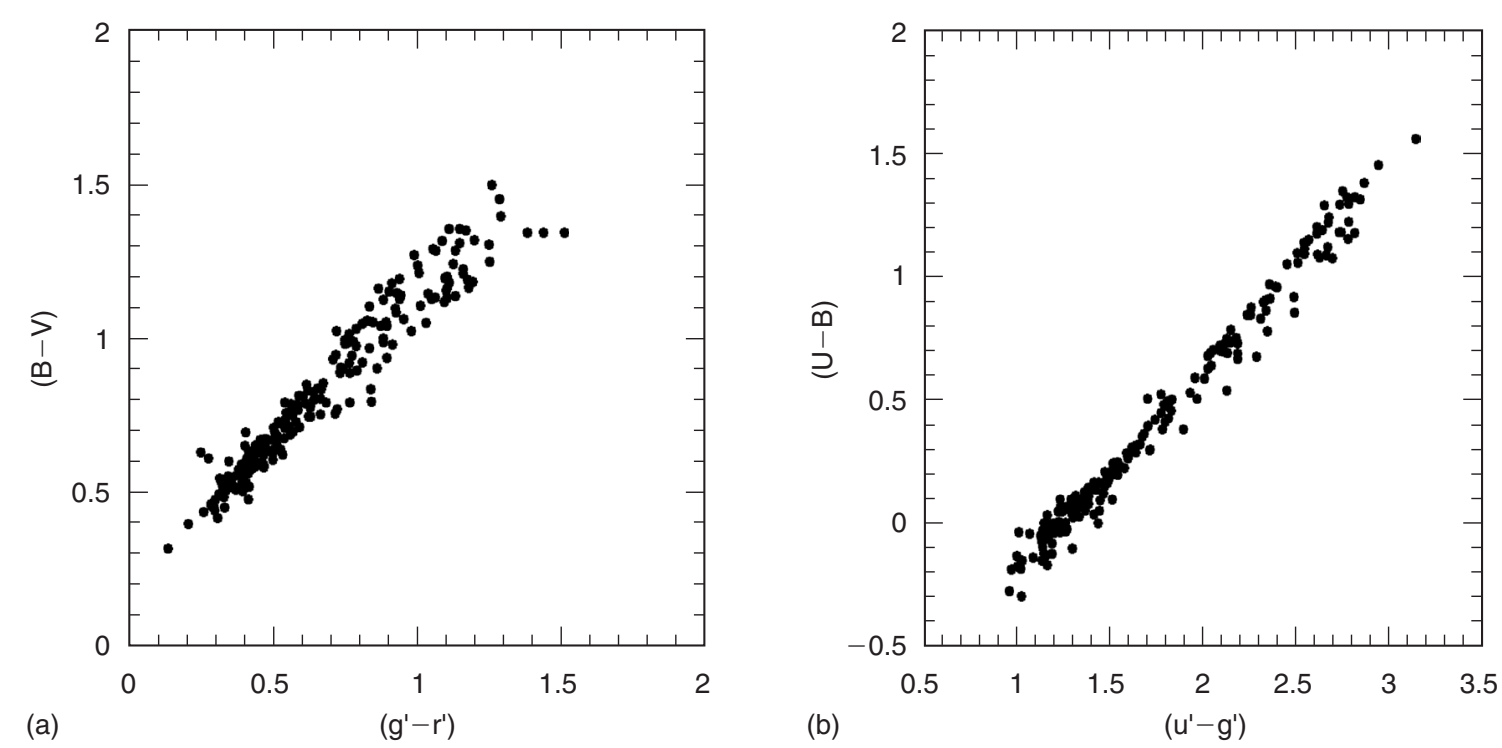

Figure 2 (a) $\left(g^{\prime}-r^{\prime}\right)$ versus $(B-V)$ and (b) $\left(u^{\prime}-g^{\prime}\right)$ versus $(U-B)$ for 224 stars. The 27 stars at extreme locations in Figure 1 are excluded from the sample.

for comparison purpose:

$$
\begin{aligned}
g^{\prime}-r^{\prime} & =1.05(B-V)-0.23 \\
u^{\prime}-g^{\prime} & =1.38(U-B)+1.14
\end{aligned}
$$

It seems that the main difference between two sets of equations is for $\left(u^{\prime}-g^{\prime}\right)$, which is sensitive to metallicity and therefore results in different absolute magnitudes for the same $\left(g^{\prime}-r^{\prime}\right)$ colour index.

To illustrate the difference between the two colour transformations, we applied each set of transformations to the $U B V$ measures and compared the predicted $\left(u^{\prime}-g^{\prime}\right)$ and $\left(g^{\prime}-r^{\prime}\right)$ colours to the actual measures from CASU
INT Wide Field Survey (Figure 3). The mean of the differences between the evaluated $\left(u^{\prime}-g^{\prime}\right)$ and the original ones is $\left\langle\Delta\left(u^{\prime}-g^{\prime}\right)\right\rangle=0.000 \mathrm{mag}$ for the new colour transformation and $\left\langle\Delta\left(u^{\prime}-g^{\prime}\right)\right\rangle=0.044 \mathrm{mag}$ for the one of Fukugita et al. (1996). The corresponding standard deviations are $s= \pm 0.017 \mathrm{mag}$ and $s= \pm 0.104 \mathrm{mag}$, respectively.

To demonstrate the superiority of our new transformations, we illustrate their impact on particular application - the derivation of photometry-based metallicity estimates. We (Karaali et al. 2003) estimated abundances based on the $\delta_{0.4}$ measure. A star of $\delta_{0.4}=0.25$ in the new transformation would have $\delta_{0.4}=0.294$ in the old 

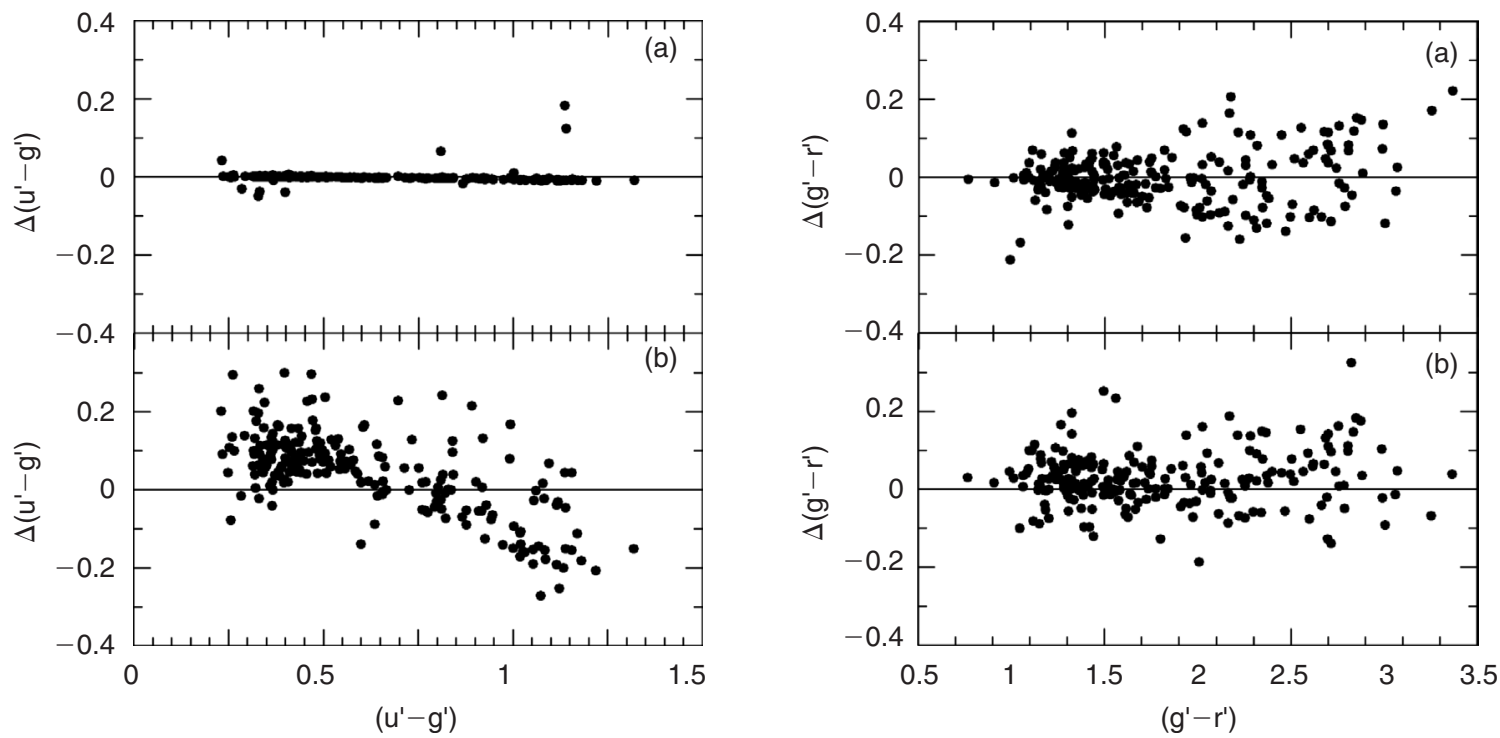

Figure 3 Comparison of the predicted (left) $\left(u^{\prime}-g^{\prime}\right)$ and (right) $\left(g^{\prime}-r^{\prime}\right)$ colours with the actual measures for (a) for the new colour transformations and (b) the colour transformations of Fukugita et al. (1996).

transformation. Using the metallicity calibration given in Karaali et al. (2003),

$$
[\mathrm{Fe} / \mathrm{H}]=0.10-2.00 \delta_{0.4}-12.64 \delta_{0.4}^{2}+11.43 \delta_{0.4}^{3}
$$

one obtains the metallicities $[\mathrm{Fe} / \mathrm{H}]=-1.01$ and $[\mathrm{Fe} / \mathrm{H}]=$ -1.29 dex, for $\delta_{0.4}=0.25$ and $\delta_{0.4}=0.294$ respectively.

Additionally, the standard deviation $s= \pm 0.104 \mathrm{mag}$ is roughly six times larger than $s= \pm 0.017$ mag. That is, the colour transformations of Fukugita et al. (1996) causes a larger dispersion relative to the new ones. All these differences cited here affect also the evaluation of absolute magnitudes and hence the distance to a star.

The evaluated $\left(g^{\prime}-r^{\prime}\right)$ colour indices by means of two sets of colour transformations are not as different as the $\left(u^{\prime}-g^{\prime}\right)$ ones. The mean of the differences between the evaluated $\left(g^{\prime}-r^{\prime}\right)$ and the original ones is $\left\langle\Delta\left(g^{\prime}-r^{\prime}\right)\right\rangle=0.000 \mathrm{mag}$ for Equation (2), and $\left\langle\Delta\left(g^{\prime}-r^{\prime}\right)\right\rangle=0.027 \mathrm{mag}$ for Equation (3). Although there is a small difference between these means, the corresponding standard deviations are almost equal, i.e. $s= \pm 0.0700 \mathrm{mag}$ and $s= \pm 0.0701 \mathrm{mag}$ for the new and old colour transformations, respectively. Such differences in $\left(g^{\prime}-r^{\prime}\right)$ do not affect either the metallicity or the absolute magnitude estimation considerably.

\section{Revised Metallicity Calibration}

We used the procedure given in our earlier paper (Karaali et al. 2003) for revising the metallicity calibration. The only difference is between the different colour transformations used. Let us write Equation (2) for two stars with the same $(B-V)$ (equivalently, $\left.\left(g^{\prime}-r^{\prime}\right)\right)$, i.e. for a Hyades star $(\mathrm{H})$ and for a star $(\star)$ whose UV-excess is normalised:

$$
\begin{aligned}
\left(u^{\prime}-g^{\prime}\right)_{\mathrm{H}} & =0.779(U-B)_{\mathrm{H}}+0.755(B-V)+0.801 \\
\left(u^{\prime}-g^{\prime}\right)_{\star} & =0.779(U-B)_{\star}+0.755(B-V)+0.801
\end{aligned}
$$

Then, the UV-excess for the star in question, relative to the Hyades star, is

$\left(u^{\prime}-g^{\prime}\right)_{\mathrm{H}}-\left(u^{\prime}-g^{\prime}\right)_{\star}=0.779\left[(U-B)_{\mathrm{H}}-(U-B)_{\star}\right]$

or, in standard notation,

$$
\delta\left(u^{\prime}-g^{\prime}\right)=0.779 \delta(U-B)
$$

The $(U-B)$ colour index of a Hyades star with $(B-V)=0.6 \mathrm{mag}$ is $(U-B)_{\mathrm{H}}=0.13 \mathrm{mag}$ (Sandage 1969), and Equation (2) transforms them to $\left(g^{\prime}-r^{\prime}\right)=$ $0.43 \mathrm{mag}$. If we apply Equation (7) to a star with $(B-V)=0.6 \mathrm{mag}$, we obtain

$$
\delta\left(u^{\prime}-g^{\prime}\right)_{0.43}=0.779 \delta(U-B)_{0.6}
$$

for the relation between the normalised UV excesses in $U B V$ and the Sloan systems. From this Equation we obtain

$$
\delta(U-B)_{0.6}=1.284 \delta\left(u^{\prime}-g^{\prime}\right)_{0.43}
$$

which yields a revised metallicity calibration for the Sloan photometry by its substitution in

$$
[\mathrm{Fe} / \mathrm{H}]=0.10-2.76 \delta_{0.6}-24.04 \delta_{0.6}^{2}+30.00 \delta_{0.6}^{3}
$$

which covers a large range of metallicity, i.e. $-2.75 \leq$ $[\mathrm{Fe} / \mathrm{H}] \leq 0.2 \mathrm{dex}$ (Karaali et al. 2003). Hence, the revised metallicity calibration for the Sloan photometry is obtained as follows:

$$
[\mathrm{Fe} / \mathrm{H}]=0.10-3.54 \delta_{0.43}-39.63 \delta_{0.43}^{2}+63.51 \delta_{0.43}^{3}
$$

We tested this calibration by comparison the metallicities for 155 stars, whose metal abundances are in the 
Table 1. Numerical values for the coefficients of Equation (13) as a function of $(B-V)$ colour index, transferred from Table 4 of Karaali et al. (2003)

\begin{tabular}{lrrrr}
\hline$(B-V)_{0}$ & \multicolumn{1}{c}{$b_{3}$} & \multicolumn{1}{c}{$b_{2}$} & $b_{1}$ & \multicolumn{1}{c}{$b_{0}$} \\
\hline$(0.3-0.4]$ & -32.1800 & 15.9370 & 1.7350 & -0.0177 \\
$(0.4-0.5]$ & -15.3820 & 3.7188 & 4.4850 & 0.0022 \\
$(0.5-0.6]$ & 3.9109 & -4.8075 & 5.3847 & 0.0134 \\
$(0.6-0.7]$ & -11.1700 & -0.3015 & 5.0281 & 0.0153 \\
$(0.7-0.8]$ & 0.1049 & -3.6157 & 4.6196 & -0.0144 \\
$(0.8-0.9]$ & -22.5350 & 0.1109 & 3.4469 & -0.0203 \\
$(0.9-1.0]$ & -24.9710 & 7.2916 & 2.0269 & 0.0051 \\
$(1.0-1.1]$ & -7.4029 & 4.2761 & 1.2638 & -0.0047 \\
\hline
\end{tabular}

range $-3.0 \leq[\mathrm{Fe} / \mathrm{H}] \leq 0.2$ dex, as estimated using Equations (10) and (11). The correlation is rather high, i.e. $[\mathrm{Fe} / \mathrm{H}]_{U B V}=0.9998[\mathrm{Fe} / \mathrm{H}]_{\text {Sloan }}-0.006$, confirming the procedure used for derivation of the revised metallicity calibration for the Sloan photometry.

\section{Revised Equations for the Sloan Photometry for Photometric Parallax Estimation}

\subsection{The Procedure}

We recall the procedure used in our earlier paper (Karaali et al. 2003) for photometric parallax estimation for the $U B V$ photometry as follows: We separated the stars into eight $(B-V)$ colour index intervals, $(0.3-0.4],(0.4-0.5]$, (0.5-0.6], (0.6-0.7], (0.7-0.8], (0.8-0.9], (0.9-1.0], and (1.0-1.1], and used the normalised equation

$$
\begin{aligned}
& M_{V}^{\mathrm{H}}(\text { nor }) \\
& \quad=-2.1328(B-V)^{2}+8.6803(B-V)+0.305
\end{aligned}
$$

for the fiducial main-sequence of Hyades as a standard main-sequence, and derived the metallicity dependent offset

$$
\Delta M_{V}^{\mathrm{H}}(\text { nor })=b_{3} \delta_{0.6}^{3}+b_{2} \delta_{0.6}^{2}+b_{1} \delta_{0.6}+b_{0}
$$

for each $(B-V)$ interval (we used the symbols in Equation (13) in a manner identical to the earlier paper for consistency). The coefficients which were evaluated by 1236 stars are transferred here into Table 1 . Thus, the $M_{V}(\star)$ absolute magnitude of a star with given $(B-V)$ colour index cited above can be estimated via $\Delta M_{V}^{\mathrm{H}}($ nor $)=M_{V}(\star)-M_{V}^{\mathrm{H}}($ nor $)$, provided that its $(U-B)$ colour index is available for $\delta_{0.6}$-normalised UV-excess evaluation.

\subsection{Photometric Parallaxes}

We need to transform Equations (12) and (13) into the Sloan data. First we start with Equation (12). Unfortunately, this is not as easy as in the case of colour transformations of Fukugita et al. (1996), for $(B-V)$ is not only the function of $\left(g^{\prime}-r^{\prime}\right)$ but also $\left(u^{\prime}-g^{\prime}\right)$. However, the inverse colour transformations in Equation (2)
Table 2. Numerical values for the coefficients of Equation (16) as a function of $\left(g^{\prime}-r^{\prime}\right)$ colour index. The colour index intervals correspond to the $(B-V)$ intervals in the first column of Table 1

\begin{tabular}{lrrrr}
\hline$\left(g^{\prime}-r^{\prime}\right)_{0}$ & \multicolumn{1}{c}{$c_{3}$} & \multicolumn{1}{c}{$c_{2}$} & \multicolumn{1}{c}{$c_{1}$} & \multicolumn{1}{c}{$c_{0}$} \\
\hline$(0.12-0.22]$ & -32.1800 & 15.9370 & 1.7350 & -0.0177 \\
$(0.22-0.32]$ & -15.3820 & 3.7188 & 4.4850 & 0.0022 \\
$(0.32-0.43]$ & 3.9109 & -4.8075 & 5.3847 & 0.0134 \\
$(0.43-0.53]$ & -11.1700 & -0.3015 & 5.0281 & 0.0153 \\
$(0.53-0.64]$ & 0.1049 & -3.6157 & 4.6196 & -0.0144 \\
$(0.64-0.74]$ & -22.5350 & 0.1109 & 3.4469 & -0.0203 \\
$(0.74-0.85]$ & -24.9710 & 7.2916 & 2.0269 & 0.0051 \\
$(0.85-0.95]$ & -7.4029 & 4.2761 & 1.2638 & -0.0047 \\
\hline
\end{tabular}

can be obtained by mathematical calculations. The one for $(B-V)$ that will be used in Equation (12) is as follows:

$$
(B-V)=0.992\left(g^{\prime}-r^{\prime}\right)-0.0199\left(u^{\prime}-g^{\prime}\right)+0.202
$$

If we replace the equivalence of $(B-V)$ in Equation (14) into Equation (12) and simplify it, we find the normalised colour-magnitude equation for the Hyades main-sequence as in the following:

$$
\begin{aligned}
M_{g^{\prime}}^{\mathrm{H}}(\text { nor })= & -2.0987\left(g^{\prime}-r^{\prime}\right)^{2}-0.0008\left(u^{\prime}-g^{\prime}\right)^{2} \\
& +0.0842\left(g^{\prime}-r^{\prime}\right)\left(u^{\prime}-g^{\prime}\right)+7.7557\left(g^{\prime}-r^{\prime}\right) \\
& -0.1556\left(u^{\prime}-g^{\prime}\right)+1.9714
\end{aligned}
$$

The transformation of Equation (13) from $U B V$ to the Sloan photometry is simple. It can be done by replacing the equivalence of $\delta_{0.6}$ in Equation (9) into Equation (13). The result is as follows:

$$
\Delta M_{g^{\prime}}^{\mathrm{H}}(\text { nor })=c_{3} \delta_{0.43}^{3}+c_{2} \delta_{0.43}^{2}+c_{1} \delta_{0.43}+c_{0}
$$

where $c_{3}=(1.284)^{3} b_{3}, c_{2}=(1.284)^{2} b_{2}, c_{1}=(1.284) b_{1}$, and $c_{0}=b_{0}$. The numerical data for $c_{i}(i=0,1,2,3)$ are given in Table 2.

As mentioned in Section 2, the mean of the differences between predicted $\left(u^{\prime}-g^{\prime}\right)$ and the actual $\left(u^{\prime}-g^{\prime}\right)$, from the CASU INT Wide Field Survey, is $\left\langle\Delta\left(u^{\prime}-g^{\prime}\right)\right\rangle=0.000 \mathrm{mag}$ for the new colour transformations and $\left\langle\Delta\left(u^{\prime}-g^{\prime}\right)\right\rangle=0.044 \mathrm{mag}$ for the one of Fukugita et al. (1996). An excess of $0.044 \mathrm{mag}$ in $\delta_{0.4}$ corresponds to an excess in absolute magnitude difference of $\sim 0.1 \mathrm{mag}$ which can be confirmed as in Section 2 . Actually, the evaluation of $\Delta M_{g^{\prime}}^{\mathrm{H}}$ (nor) by means of equation (20) in Karaali et al. (2003) for $\delta_{0.4}=0.294$ mag gives an excess between 0.07 and $0.14 \mathrm{mag}$ in absolute magnitude difference relative to $\delta_{0.4}=0.25 \mathrm{mag}$, depending on the $\left(g^{\prime}-r^{\prime}\right)$ colour index of the star. Hence, we can say that the revised equations for the photometric parallax estimation improve the absolute magnitudes by at least $\sim 0.1$ mag.

We compared the absolute magnitudes for the sample mentioned in Section 3, except three stars, derived by means of two systems, i.e. $U B V$ and Sloan. Stars excluded 


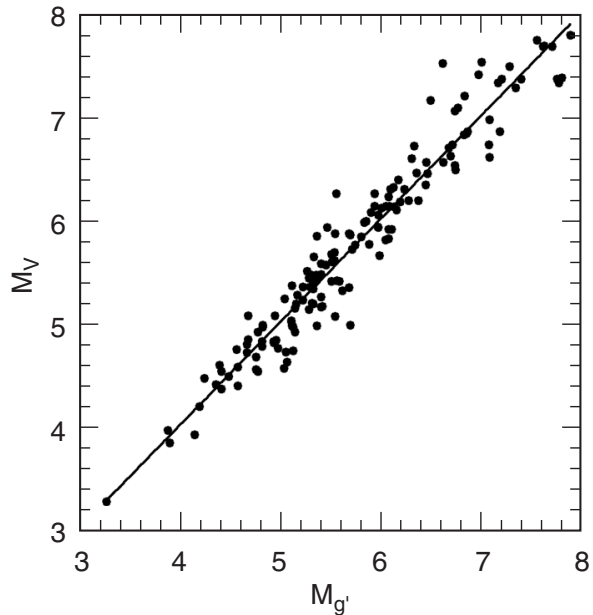

Figure 4 Comparison of the absolute magnitudes $M_{g^{\prime}}$ and $M_{V}$ derived by means of the procedure given in the text

from the sample are relatively faint and have standard errors in $\left(u^{\prime}-g^{\prime}\right)$ and $\left(g^{\prime}-r^{\prime}\right)$ larger than $0.1 \mathrm{mag}$. The mentioned comparison is given in Figure 4. Although we don't expect one to one correspondence due to different bands in two systems, the relation is rather high, again confirming our procedure, i.e. $M_{V}=0.9972 M_{g^{\prime}}-0.0460$.

\section{Conclusions}

Although the new procedure given in Karaali et al. (2003) provides significant improved photometric parallaxes with respect to the work of Laird, Carney, \& Latham (1988), it is compromised by the colour transformations of Fukugita et al. (1996). Hence, we evaluated new colour transformations for the Sloan photometry by 224 standards and used them to revise both the equations for photometric parallax estimation and metallicity calibration. There is a high correlation for metallicities and absolute magnitudes derived for two systems, $U B V$ and Sloan, by means of the revised calibrations. This process improves the metallicity up to $0.3 \mathrm{dex}$ (Section 2) and absolute magnitude at least $0.1 \mathrm{mag}$ (Section 4). The improvements will provide better results in the works of Galactic structure related with the metallicity gradient and space density functions.

We should mention that the equations for Sloan photometric parallax and $[\mathrm{Fe} / \mathrm{H}]$ would be significantly better if an analysis were made purely from Sloan data. However, our aim is to confirm the improved of the conversion of Sloan photometry to broad $U B V$ indices.

\section{Acknowledgement}

We thank the anonymous referees for their suggestions to put the text into a better and more understandable form.

\section{References}

Buser, R., 1978, A\&A, 62, 411

Chen, B., Stoughton, C., Smith, J. A., Uomoto, A., Pier, J. R., Yanny, B., Ivezic, Z., York, D. G., Anderson, J. E., \& 7 coauthors, and the SDSS Collaboration 2001, ApJ, 553, 184

Fukugita, M., Ichikawa, T., Gunn, J. E., Doi, M., Shimasaku, K., \& Schneider, D. P. 1996, AJ, 111, 1748

Karaali, S., Karataş, Y., Bilir, S., Ak, S. G., \& Hamzaoğlu, E. 2003, PASA, 20, 270

Laird, J. B., Carney, B. W., \& Latham, D. W. 1988, AJ, 95, 1843

Landolt, A. U. 1992, AJ, 104, 340

Siegel, M. H., Majewski, S. R., Reid, I. N., \& Thompson, I. B. 2002, ApJ, 578, 151

Smith, J. A., Tucker, D. L., Kent, S., Richmond, M. W., Fukugita, M., Ichikawa, T., Ichikawa, S., Jorgensen, A. M., \& 14 coauthors 2002, AJ, 123, 2121

Sandage, A. R. 1969, ApJ, 158, 1115 